\title{
The Effectiveness Evaluation based on a Revised FAHP Method
}

\author{
Yongfeng Ma ${ }^{1,}$, Yufei Chen 2, b, Jihui Yang ${ }^{1, ~ c}$, Junhua Jia 1, d \\ ${ }^{1}$ Changchun Vocational Institute of Technology, Changchun 130117, China \\ 2 Bengbu college, Bengbu 233050, China \\ a36261028@qq.com, b15975346@ qq.com, c74125869@ qq.com, d45615982@ qq.com
}

\begin{abstract}
The missing of the judgement value is a common issue in the effectiveness evaluation practice of the materiel support command and control. Based on the fuzzy consistency, a estimation and compensation algorithm of incomplete comparison matrix is proposed, and it revise the tradition FAHP methods, then the effectiveness evaluation method and process of the materiel support command and control based on the revised method are discussed, finally, a case which takes the command and control system of a certain unit as the research object is proceeded.
\end{abstract}

Keywords: support command and control; effectiveness evaluation; analytic hierarchy process; incomplete compassion matrix.

\section{Introduction}

Due to better combine the subjectivity and the objectivity, qualitative and quantitative, analytic hierarchy process (AHP) have achieved good effect in the effectiveness evaluation practice of the system, but the AHP also exposed some problems while using, for example, it is not effective to handle imprecise judgment value and uncertainty, therefore, some scholars put forward the concept of fuzzy analytic hierarchy process (FAHP), in order to improve the applicability of AHP. The main research in this area includes: Buckley put forward the concept of fuzzy matrix, and defined the concept of fuzzy matrix consistency; Holland scholar Van Loargoven first proposed a method by using a triangular fuzzy number to represent fuzzy comparison judgment matrix, and sorted the elements.

In the effectiveness evaluation practice of the materiel support command and control which based on AHP, evaluation experts may not know about the relative importance between some indexes, at this time, if the experts are required to graded the relative weight value mechanically, it will reduce the scientific and objective of the evaluation, to solve this problem, the experts are allowed to use missing values to represent the situation of unawareness, dimness, unacquaintance, which lead to the problem of incomplete information of FAHP, therefore, the effectiveness evaluation method of the materiel support command and control which based on incomplete information of FAHP should be researched.

From the published literature, in the research of FAHP which based on triangular fuzzy number, the research of experts' judgment missing values problem is lacked, so this article proposes a modified algorithm to compensate for incomplete information based on the FAHP, and the algorithm was applied to equipment support effectiveness evaluation.

\section{Fuzzy comparison judgment matrix and the definition of its consistency}

Fuzzy comparison judgment matrix is the important foundation of fuzzy AHP model proposed by Buckley and Van Loargoven, the following is the definition of the main concepts.

Definition 1: The triangular fuzzy number. A triangular fuzzy number can be defined as three values of $1, \mathrm{~m}, \mathrm{u}$, the three tuple $\tilde{R} \mathrm{ij}=(1, \mathrm{~m}, \mathrm{u})$, which describes the fuzzy when deciding and judging. Common operations of triangular fuzzy number is addition, multiplication, division, tuple, specifically defined as: let $\mathrm{M} 1=(11, \mathrm{~m} 1, \mathrm{u} 1), \mathrm{M} 2=(12, \mathrm{~m} 2, \mathrm{u} 2)$, so $\mathrm{M} 1 \oplus \mathrm{M} 2=(11+12, \mathrm{~m} 1+\mathrm{m} 2, \mathrm{u} 1+\mathrm{u} 2)$, $\mathrm{M} 1 \otimes \mathrm{M} 2=(11 \cdot 12, \mathrm{~m} 1 \cdot \mathrm{m} 2, \mathrm{u} 1 \cdot \mathrm{u} 2), \mathrm{M} 1 / \mathrm{M} 2=(11 \div 12, \mathrm{~m} 1 \div \mathrm{m} 2, \mathrm{u} 1 \div \mathrm{u} 2)$, there are the tuple operations: $(\mathrm{M}) \mathrm{L}=\mathrm{l},(\mathrm{M}) \mathrm{M}=\mathrm{m}$ and(M) $\mathrm{U}=\mathrm{u}$. 
Definition 2: Fuzzy comparison judgment matrix. By the form of pairwise comparison to determine the relative importance of decision between the indexes, and the triangular fuzzy numbers to express, to form the judgment matrix called the fuzzy comparative judgment matrix, can be expressed as $\tilde{M} \cdot \tilde{M}=[\tilde{R} \mathrm{ij}] \mathrm{n} \times \mathrm{n}$

And $\tilde{M}$ ij is a triangular fuzzy number, $\mathrm{n}$ is the order of matrix, and the number of index too. Which is similar to the traditional comparative judgment matrix, fuzzy comparison judgment matrix is reciprocal matrix, can be called the fuzzy reciprocal matrix.

Definition 3: fuzzy consistency. To fuzzy comparison judgment matrix, $\tilde{M}=[\tilde{R} \mathrm{ij}] \mathrm{n} \times \mathrm{n}$, if $\tilde{R} \mathrm{ij} \otimes \tilde{R} \mathrm{jk} \approx \tilde{R} \mathrm{ik}, \forall \mathrm{i}, \mathrm{j}, \mathrm{k}=1,2, \ldots, \mathrm{n}$, the fuzzy positive reciprocal matrix will have fuzzy consistency.

\section{Incomplete information and its compensation in FAHP}

In traditional AHP, each element of the judgment matrix must be completely given by decision makers, can be established a pairwise comparison matrix, but Harker believes that when they do not known the questionnaire, these is no necessary to answer, so he proposed the concept of incomplete pairwise comparison matrix, to truly reflected the objectivity of the fact, it must be used some methods to deal with, when there is a situation of incomplete information in the judgment matrix.

As for a traditional complete information judgment matrix A, assumed that its elements are aij, if it achieves the requirements of consistency, then for any $\mathrm{k}$ :

$a_{i j}=a_{i k} / a_{j k}$ or $a_{i j}=a_{k j} / a_{k i}(i, j, k=1,2, \ldots, m)$

That is to say, if aij is a missing judgment value, it should be given to determine the value of a variety of data on the value of the missing of contact from the consistent point of view, so, the missing value can be estimate from these.

We can use the arithmetic average value compensation of the known judgement value as the estimated value or missing value of the missing value.

$$
\mathrm{a}_{\mathrm{ij}}=\left[\coprod_{k=1}^{\mathrm{m}} a_{i k} / a_{j k}\right]^{1 / m} \text { or } \mathrm{a}_{\mathrm{ij}}=\left[\coprod_{k=1}^{\mathrm{m}} a_{k j} / a_{k i}\right]^{1 / m}
$$

$a_{i k}, a_{j k}, a_{k j}$, $a_{k i}$ are known judgement value, $n$ is the order number of a matrix, $m$ is the number of rows or columns of the available compensation data. The former of the formula is compensated by row, and the compensation information sources from the elements in $\mathrm{i}$ and $\mathrm{j}$ row, the latter is compensated by column, and the compensation information sources from the elements in $\mathrm{i}$ and $\mathrm{j}$ column.

Then, extended the above method to fuzzy judgment matrix. For a complete information fuzzy judgment matrix $\tilde{M}=\left[\tilde{R}_{\mathrm{ij}}\right]_{\mathrm{n} \times \mathrm{n}}$, as previously shown, if it achieves the requirements of fuzzy consistency, then for any $\mathrm{k}$ :

$$
\tilde{R}_{\mathrm{ij}} \approx \tilde{R}_{\mathrm{ik}} / \tilde{R}_{\mathrm{jk}}, \forall \mathrm{i}, \mathrm{j}, \mathrm{k}=1,2, \ldots, \mathrm{n} \text { or } \tilde{R}_{\mathrm{ij}} \approx \tilde{R}_{\mathrm{kj}} / \tilde{R}_{\mathrm{ki}}, \forall \mathrm{i}, \mathrm{j}, \mathrm{k}=1,2, \ldots, \mathrm{n}
$$

According to the relationships between elements of fuzzy consistent judgment matrix, it can be given compensation algorithm of the missing values of fuzzy judgment matrix.

Set a fuzzy judgment matrix $\tilde{M}=\left[\tilde{R}_{\mathrm{ij}}\right]_{\mathrm{n} \times \mathrm{n}}$, which can be decomposed into three matrices $\mathrm{L}, \mathrm{M}$ and $\mathrm{U}$, and L, M, U elements corresponding to the first, second, third tuple of the corresponding position in the fuzzy three tuple of $\tilde{M}$, i.e.:

$$
\begin{aligned}
& \mathrm{L}_{\mathrm{ij}}=\left(\tilde{R}_{\mathrm{ij}}\right)_{\mathrm{L}}, \forall \mathrm{i}, \mathrm{j}=1,2, \ldots, \mathrm{n} \\
& \mathrm{M}_{\mathrm{ij}}=\left(\tilde{R}_{\mathrm{ij}}\right)_{\mathrm{M}}, \forall \mathrm{i}, \mathrm{j}=1,2, \ldots, \mathrm{n} \\
& \mathrm{U}_{\mathrm{ij}}=\left(\tilde{R}_{\mathrm{ij}}\right)_{\mathrm{U}}, \forall \mathrm{i}, \mathrm{j}=1,2, \ldots, \mathrm{n}
\end{aligned}
$$

Let us compensate for the missing values of $\mathrm{L}, \mathrm{M}$ and $\mathrm{U}$. The emergence of a missing value means the weight of corresponding indexes are uncertain or unknown to the decision makers, therefore, when using the arithmetic average value to compensate, we should further enlarged the uncertainty of the compensated triangular fuzzy number, that is to say, for $L$ and $U$, can be zoom in and zoom out certain coefficient $\mathrm{z}$ on the compensated arithmetic average value, generally, $\mathrm{z}$ is $2.1 \sim 2.4$, L uses a 
compensation arithmetic average value divided by $z, U$ uses a compensation arithmetic average value multiplied by z. Specifically, the compensation formula of L, M and $U$ is in type (1).

After the L, M and $U$ were compensated for the missing values, we can reorganized a fuzzy matrix by $\mathrm{L}, \mathrm{M}$ and $\mathrm{U}$, so the fuzzy matrix is the revised fuzzy matrix.

\section{Examples of revised algorithm and analysis}

Set there is a incomplete fuzzy judgment matrix in the following, as shown in Table 1.

For this incomplete fuzzy judgment matrix, we divide it into three matrix, $\mathrm{L}, \mathrm{M}$ and $\mathrm{U}$, that $\mathrm{M}$ is:

$$
\mathrm{M}=\left[\begin{array}{cccc}
1 & 5 & 1 / x & 3.64 \\
0.2 & 1 & 0.41 & 0.74 \\
x & 2.53 & 1 & 1.84 \\
0.28 & 1.35 & 0.54 & 1
\end{array}\right]
$$

Let us compensate the $\mathrm{M}$ by Formula (1), according to the row compensation and the column compensation.

Compensation on row: (the points in Row i divided by the $\mathrm{j}$, and then get the arithmetic average)

$\mathrm{x}=\left[\coprod_{k=1}^{2} a_{i \mathrm{k}} / a_{j k}\right]^{1 / 2}=0.506$;

Can also be compensated on column, the answer of $\mathrm{x}$ is 0.503 .

In the same way, $L$ and $U$ can be compensated respectively, when $L$ and $U$ are compensated, it can get amplification coefficient $\mathrm{z}=1.3$, the answer is:

Compensation value of $\mathrm{L}$ (compensation on row):

$\mathrm{x}=\left[\coprod_{k=1}^{2} a_{i \mathrm{k}} / a_{j k}\right]^{1 / 2} \div \mathrm{z}=0.381$;

Compensation value of $U$ (compensation on row):

$\mathrm{x}=\left[\coprod_{k=1}^{2} a_{\mathrm{kj}} / a_{k i}\right]^{1 / 2} \times \mathrm{Z}=0.381$;

When L, M and U are compensated, it can be reorganized a fuzzy matrix, as shown in table 2 .

Table 1 One example of incomplete fuzzy judgment matrix.

\begin{tabular}{cccc}
\hline$(1,1,1)$ & $(4.2,5,5.8)$ & $? ? ?$ & $(2.53,3.64,4.77)$ \\
\hline$(0.17,0.2,0.24)$ & $(1,1,1)$ & $(0.28,0.41,0.61)$ & $(0.68,0.74,0.82)$ \\
$? ? ?$ & $(1.98,2.53,2.99)$ & $(1,1,1)$ & $(1.32 .1 .84,2.12)$ \\
$(0.21,0.28,0.4)$ & $(1.11,1.35,1.99)$ & $(0.38,0.54,0.63)$ & $(1,1,1)$ \\
\hline
\end{tabular}

Table 2 Complete fuzzy judgment matrix after modified compensation

\begin{tabular}{cccc}
\hline$(1,1,1)$ & $(4.2,5,5.8)$ & $(1.608,1.976,2.625)$ & $(2.53,3.64,4.77)$ \\
\hline$(0.17,0.2,0.24)$ & $(1,1,1)$ & $(0.28,0.41,0.61)$ & $(0.68,0.74,0.82)$ \\
$(0.381,0.506,0.622)$ & $(1.98,2.53,2.99)$ & $(1,1,1)$ & $(1.32 .1 .84,2.12)$ \\
$(0.21,0.28,0.4)$ & $(1.11,1.35,1.99)$ & $(0.38,0.54,0.63)$ & $(1,1,1)$ \\
\hline
\end{tabular}

The revised method of FAHP based on incomplete information compensation has the following characteristics: (1) the method use the consistency of fuzzy judgement matrix, which has a strict mathematical foundation; (2) the method is simple and feasible in computation, the main procedure is point divide operation in a row or column, convenient for manual or computer operation; (3) a lot of examples shows that, the answer has more consistency by compensation on row and compensation on column, that proved the scientific of the method; (4) the method can be proper amplification the compensation interval of $\mathrm{L}$ and $\mathrm{U}$, in accordance with decision psychology of the people, because 
policymakers uncertain judgment value, fuzzy values tend to be larger; (5) by using this method, greatly increase the humanized and efficiency of FAHP method, so that the decision makers can be more reasonable, more active, more fully to tap their own judgment.

\section{Conclusion}

This article on the use of fuzzy judgment matrix with fuzzy consistency shows the estimation of evaluation value under the incomplete information situation and the compensation method, make full use of the method, it can easily solve the evaluation questionnaire constraints and limitations to evaluation experts, so humanization level which AHP method used in the effectiveness evaluation of materiel support command and control greatly enhanced.

\section{Reference:}

[1] Li Yimin, Luo Aimin, Luo Xueshan. An improved AHP method and its effectiveness analysis of G3I system in the application[J]. Command control and simulation, 2006,28 (4): 37-40.

[2] Buckley J J. Fuzzy Hierarchical Analysis[J]. Fuzzy Sets and Systems,1985,(17):233-247

[3] Laarhoven P J M, Pedrycz W. A Fuzzy Extension of Saaty s Priority Theorem[J]. Fuzzy Sets and Systeme,1983,(11):229-241

[4] Kazutomo N. Estimation of Unknown Comparisons in Incomplete AHP and it's Compensation[R]. Report of the Research Institute of Industrial Technology. Nihon University. 2005.

[5] Harker P T Incomplete Pairwise Comparisons in the Analytic Hierarchy Process[J]. Mathematical Modelling,1987(9):837-848.

[6] Li Youwen, Hao Qizong. Adjacent positive reciprocal matrix perturbation effects on the weight vector [J]. Journal of Beijing Institute of Technology, 1999,19 (3): 275-279 